\title{
Vigilancia Paramétrica de la Bilinealidad de Transmisores de Presión por Análisis de Ruido
}

\author{
Cristina Montalvo ${ }^{1}$, Miguel Balbás ${ }^{1}$, Juan Blázquez ${ }^{2}$ y José M. Chicharro ${ }^{3}$ \\ (1) Universidad Politécnica de Madrid, ETSI de Minas, Grupo de Investigación AMERPREM, \\ Ríos Rosas 21, 28003 Madrid-España \\ (2) CIEMAT, Departamento de Fisión Nuclear, Avenida Complutense 22, 28040 Madrid-España \\ (3) Universidad de Castilla La Mancha, Área de Ingeniería Mecánica, ETSI Industriales, \\ Campus Universitario S/N, 13071 Ciudad Real-España
}

\begin{abstract}
Resumen
El objetivo del presente trabajo es detectar la no linealidad asociada al comportamiento de un transmisor de presión capacitivo tipo Rosemount, cuando éste sufre pérdidas de aceite. La metodología propuesta consiste en suponer un comportamiento dinámico bilineal. Se simula la respuesta tanto del transmisor como de su línea sensora a una excitación de tipo ruido blanco y se trata la señal de medida aplicando modelos autorregresivos univariantes. Se plantea la simulación introduciendo el empleo de dos familias de coeficientes autorregresivos para reproducir la bilinealidad, no considerando la ecuación estocástica asociada a la excitación por ruido blanco. A partir de los resultados se ha definido un índice de vigilancia paramétrica para complementar la medida del tiempo de respuesta del sensor, ya que dicho tiempo no es la magnitud adecuada para detectar la bilinealidad.
\end{abstract}

Palabras clave: transmisor, análisis de ruido, bilinealidad, vigilancia, ecuaciones estocásticas

\section{Parametric Surveillance of Pressure Transmitters Bilinearity through Noise Analysis}

\begin{abstract}
The aim of this work is detecting the non linearity associated to a Rosemount capacitive transmitter when it suffers oil loss syndrome. The proposed methodology to characterize the non-linearity consists of assuming bilinear dynamic behaviour. The transmitter and sensing line response to a driven white noise is simulated and the signal is treated applying univariant autoregressive models. As a new approach, the simulation is achieved introducing the use of two autoregressive coefficients families to reproduce the bilinearity, omitting the stochastic equation associated to the driven white noise. Base on the analysis of the results, a parametric surveillance index is defined to complement the measurement of the response time, as this time is not the appropriate quantity to detect the bilinearity.
\end{abstract}

Keywords: transmitter, noise analysis, bilinearity, surveillance, stochastic equations 


\section{INTRODUCCIÓN}

Diversos modelos de sensores capacitivos, tipo Rosemount, son muy utilizados en plantas nucleares, tanto en sus versiones de medida del caudal, del nivel o de la presión; son sensores de muy buena precisión y la vigilancia de su funcionamiento se realiza normalmente mediante análisis de ruido (Ortiz-Villafuerte et al., 2006). Existen varias anomalías en su funcionamiento que son fácilmente detectables en el análisis del ruido (García-Berrocal et al., 2005; Lin y Holbert, 2008); en el caso de los sensores de presión se pueden producir algunos fallos más difíciles de evidenciar: existencia de burbujas en la línea sensora o válvulas de aislamiento medio cerradas, por ejemplo. El procedimiento ordinario de vigilancia consiste en analizar el ruido para medir in situ el tiempo de respuesta (Bergdahl et al., 2003). Este valor es la base para desarrollar el mantenimiento predictivo del sensor.

La pérdida de aceite de silicona en un sensor de presión es una anomalía difícil de detectar (Glökler et al., 2000). El aceite de silicona rellena un pequeño canal que reduce la presión entre la membrana de aislamiento y la membrana sensora. Como el sensor es capacitivo, el aceite actúa también como dieléctrico influyendo en la capacidad eléctrica del sensor. Dicha pérdida de aceite provoca que el comportamiento de la membrana sensora pierda su simetría, actuando con una rigidez distinta según el signo de la sobrepresión a que esté sometida. El tiempo de respuesta del sensor no es sensible al síndrome de pérdida de aceite de silicona; la respuesta ya no es lineal, con lo que, en rigor, si se somete al sensor a una rampa de presión, el tiempo de respuesta depende del signo de la rampa. El cambio de las condiciones dinámicas del sensor produce un comportamiento bilineal. La aparición de no linealidades en los sistemas de planta, aparte de ser un indicador de avería incipiente, hace necesario implementar estrategias de compensación en los lazos de control (Vásquez y Castrillón, 2007). Se han estudiado procedimientos empíricos para lograr una detección temprana de esta anomalía, pero en este trabajo se desarrolla una justificación teórica mediante la modelización del sensor, con la posibilidad de obtener un índice de alarma que evidencie la existencia del síndrome.

\section{ANTECEDENTES}

Cuando se produce una pérdida de aceite se puede observar que el comportamiento del sensor da lugar a un desvío significativo de la medida en relación con los valores dados por otros canales redundantes, al tiempo que una disminución del nivel de ruido a la salida del sensor, dificultad para su calibración y respuesta lenta después de una parada del reactor.

Todo ello fue observado por primera vez en 1987, en transmisores de presión diferencial Rosemount 1153HD5PC. Estos transmisores fueron declarados fuera de servicio y el fabricante, tras diversas pruebas, identificó la anomalía como una fuga del aceite. Como consecuencia de este suceso algunos investigadores y el propio fabricante (Rosemount Eng. Company, 1992) emprendieron estudios para entender este síndrome y generar procedimientos de detección incipiente.

Las investigaciones experimentales demostraron que la membrana retardaba su vuelta a la posición de equilibrio después de una sobrepresión (Hashemian et al., 1993), con lo que se ocasionaba una asimetría en la vibración de la membrana.

Esta asimetría de las oscilaciones se refleja en los valores del ruido, hay una relación entre el sesgo de la distribución de amplitudes (Hashemian et al., 1993) y el valor de la pérdida.

En la Figura 1 se muestra un ejemplo característico de ruido bilineal. Sin embargo el detectar sesgo en el ruido no es suficiente para asegurar la existencia del síndrome, porque también otras anomalías pueden dar lugar al sesgo. Debe existir al mismo tiempo una disminución de la varianza.

En las pruebas en el Laboratorio se encontraron tiempos de respuesta diferentes según que la rampa fuera positiva o negativa; especialmente, en el modelo indicado, en el caso en que el punto de tarado correspondía a baja presión, las membranas vuelven a su posición de equilibrio muy lentamente en la rampa negativa, con tiempos de respuesta muy superiores a los obtenidos para la rampa positiva. 


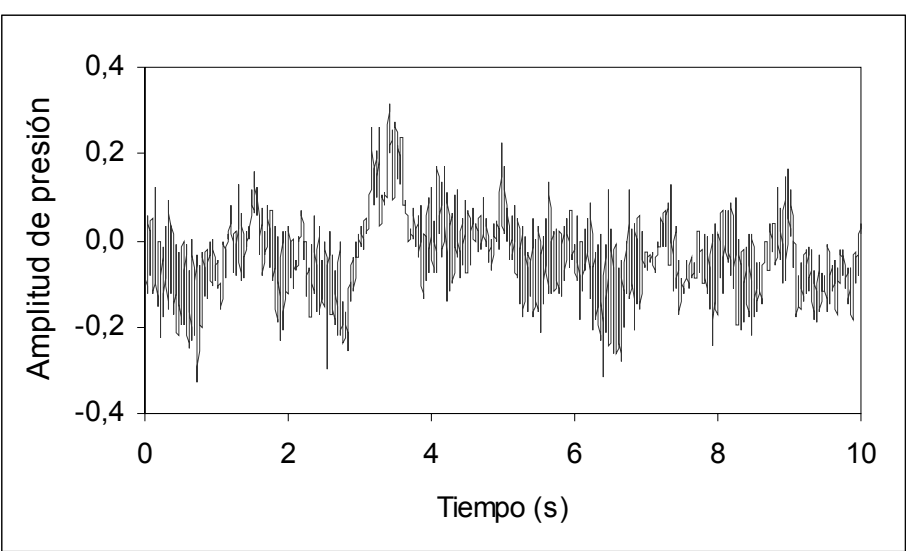

Fig. 1: Ruido típico en el síndrome de pérdida de aceite

\section{MODELIZACIÓN DEL SENSOR}

La modelización está orientada a explicar el espectro de frecuencia del ruido. Incluye a la línea sensora y al sensor (Lin y Holbert, 2009). En la región de frecuencia de interés, por debajo de los 20 $\mathrm{Hz}$, la longitud de la línea, del orden de $25 \mathrm{~m}$ y trazado irregular, es varias veces menor que la longitud de onda de propagación del sonido en el agua líquida a esta frecuencia. Este resultado permite modelizar la línea como si fuera puntual mediante una analogía eléctrica (Blázquez y Vela, 1997).

La Figura 2 muestra la analogía eléctrica propuesta. Los valores típicos de los parámetros de dicho circuito se han tomado de la literatura (Barbero et al., 2000), y se recogen en la Tabla 1.
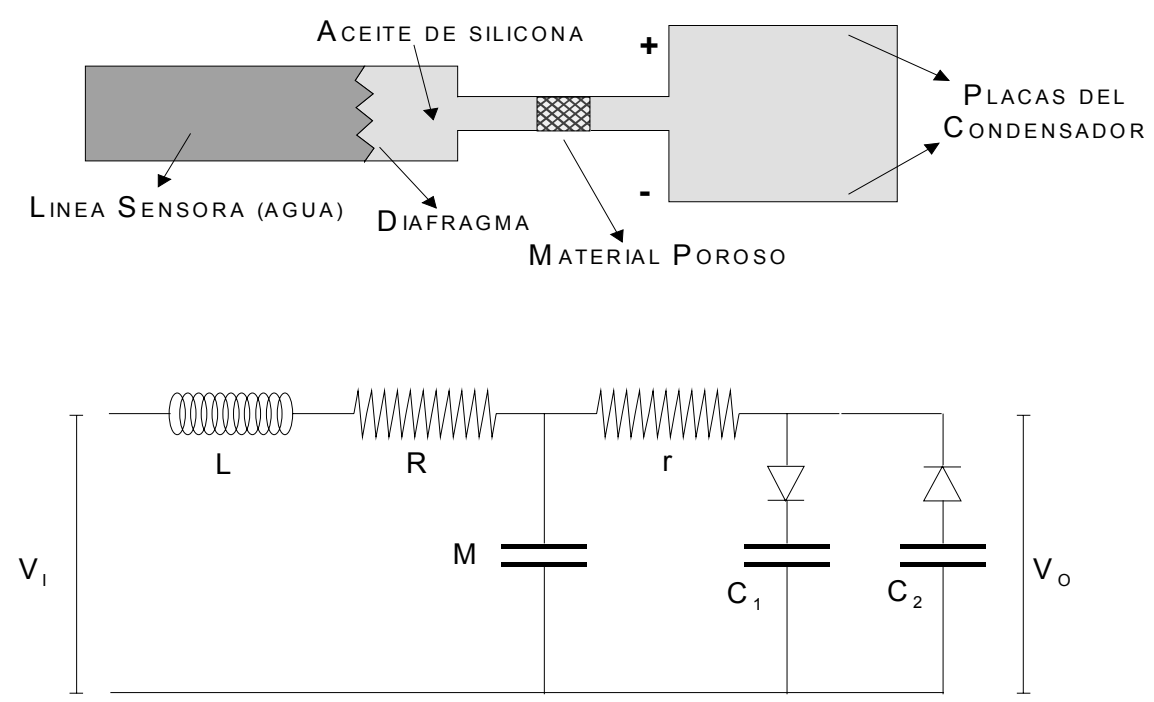

Fig. 2: Modelo físico simplificado del sensor de presión y su correspondiente eléctrico

Las características del síndrome podrían explicarse repartiendo la capacidad de la membrana sensora en dos condensadores. Cuando la presión es positiva, hay una rigidez dieléctrica normal $\left(C_{1}\right)$, y cuando es negativa, la pérdida de aceite ocasiona otra rigidez $\left(C_{2}\right)$. Para separarlas se incluyen los diodos en el modelo, que deja así de ser lineal.

La respuesta no lineal se puede modelizar empleando dos funciones de transferencia cuya forma es (Blázquez y Vela, 1997):

$$
H(s)=\frac{1 / C s}{(L s+R)\left(1+\frac{r+1 / L s}{1 / M s}\right)+r+1 / C s}
$$


Tabla 1: Valores típicos de los parámetros del sensor y la línea sensora (literatura)

\begin{tabular}{|c|c|c|}
\hline Sistema mecánico & Sistema eléctrico & $\begin{array}{c}\text { Valores típicos } \\
\text { (unidades en el SI) }\end{array}$ \\
\hline Presión & Voltaje $\left(V_{l}, V_{0}\right)$ & $\mathrm{Pa}$ \\
\hline Volumen desplazado & Intensidad & $\mathrm{m}^{3}$ \\
\hline $\begin{array}{c}\text { Inercia de la línea } \\
\text { sensora }\end{array}$ & Inductancia $(L)$ & $5,55 \times 10^{7} \mathrm{~Pa} \mathrm{~s}^{2} \mathrm{~m}^{-3}$ \\
\hline $\begin{array}{l}\text { Rozamiento en la línea } \\
\text { sensora }\end{array}$ & Resistencia $(R)$ & $2,21 \times 10^{8} \mathrm{~Pa} \mathrm{~s} \mathrm{~m}{ }^{-3}$ \\
\hline $\begin{array}{l}\text { Rigidez del diafragma } \\
\text { de aislamiento }\end{array}$ & Capacidad $(M)$ & $1,03 \times 10^{-12} \mathrm{~m}^{3} \mathrm{~Pa}^{-1}$ \\
\hline $\begin{array}{l}\text { Rozamiento en la línea } \\
\text { con aceite }\end{array}$ & Resistencia $(r)$ & $2,5 \times 10^{12} \mathrm{~Pa} \mathrm{~s} \mathrm{~m}^{-3}$ \\
\hline $\begin{array}{l}\text { Rigidez del diafragma } \\
\text { sensor }\end{array}$ & Capacidad $\left(C, C_{1}, C_{2}\right)$ & $1,61 \times 10^{-12} \mathrm{~m}^{3} \mathrm{~Pa}^{-1}$ \\
\hline
\end{tabular}

Una de las funciones de transferencia se obtiene seleccionando $C=C_{1}$ si $V_{0} \geq 0$, y la otra tomando $C=C_{2}$ si $V_{0}<0$. Esto es necesario para explicar por qué los tiempos de respuesta a rampas positivas y negativas son distintos. Las respuestas a la rampa vienen dadas por la transformada inversa de Laplace de $H(s) / s^{2}$. De aquí pueden calcularse los tiempos de respuesta para ambas rampas.

\section{RESULTADOS DEL ANÁLISIS DE RUIDO}

La primera manifestación del síndrome es la pérdida de amplitud del ruido. En la Figura 3 se observa cómo disminuye la varianza al aumentar la capacidad $C_{2}$ respecto a la de referencia $C_{1}$. Los sensores redundantes que no sufren el síndrome son la clave para identificar el primer síntoma. Es necesario también detectar la falta de simetría.

En la Figura 4 se ha representado el sesgo en función del cambio de capacidad. Es una función creciente debido a que la asimetría es manifiesta y a que la varianza es decreciente. Los sensores redundantes que no sufren el síndrome tienen un sesgo nulo.

El análisis de frecuencia es menos adecuado para la detección del síndrome. Se debe a que la línea sensora y la rigidez de la membrana aisladora, que explican la resonancia del espectro, permanecen inalterados por el síndrome, habiéndose observado solamente un pequeño corrimiento del polo real hacia la izquierda. 


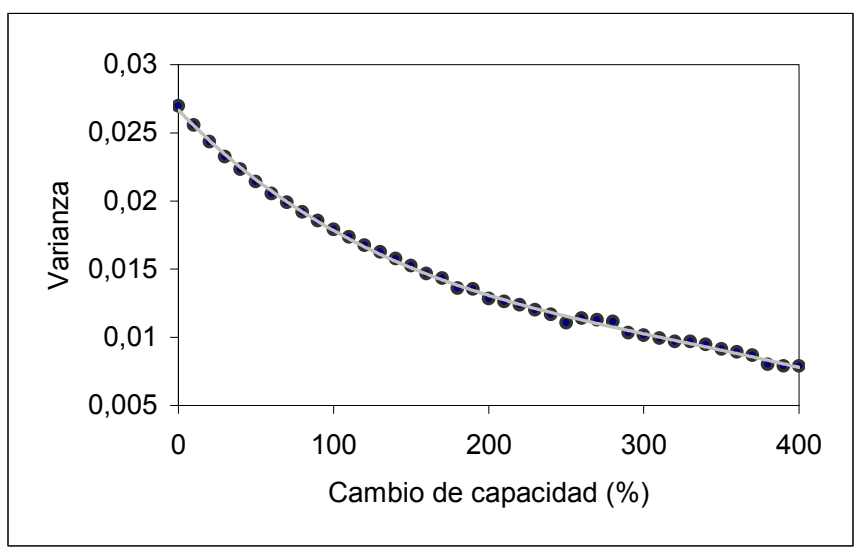

Fig. 3: Reducción de la varianza del ruido en función del cambio de capacidad $\Delta C / C_{1}=\left(C_{2}-C_{1}\right) / C_{1}$ asociado a la presencia del síndrome.

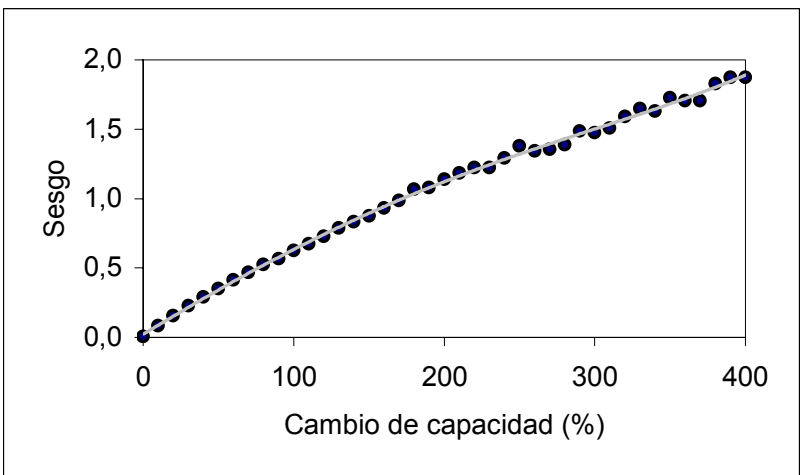

Fig. 4: Aumento del sesgo del ruido en función del cambio de capacidad $\Delta C / C_{1}=\left(C_{2}-C_{1}\right) / C_{1}$ asociado a la presencia del síndrome.

El ruido se analiza en el dominio del tiempo (Priestly, 1981). En la práctica se ajusta un modelo autorregresivo (AR) a la serie temporal de ruido. Si $x_{j}$ representa el valor del ruido en el instante $t_{j}$, entonces:

$x_{j}=\sum_{k=1}^{n} a_{k} x_{j-k}+\varepsilon_{j} ; j=0, \ldots, N$

siendo: $N$ el tamaño de la muestra; $a_{k}$, los coeficientes $A R, n$ el orden del ajuste y $\varepsilon$ el ruido blanco gaussiano de entrada. Los coeficientes $a_{k}$ se obtienen resolviendo las ecuaciones de Yule-Walker y el orden del ajuste, aplicando el criterio de Akaike al ruido residual.

Los coeficientes AR se han obtenido a partir de la función de transferencia $H(s)$ con tres pasos sucesivos: Cálculo de la respuesta al impulso (transformada inversa de Laplace de la función de transferencia) y su correspondiente función de autocorrelación, y resolución de las ecuaciones de Yule-Walker (Priestly, 1981).

El tiempo de respuesta es la base para la vigilancia de los sensores, pero en un sistema no lineal no es unívoco, por lo que, en rigor, este concepto no debería usarse. Además, aunque $\tau$ se calculase, no es el parámetro de vigilancia adecuado para detectar el síndrome de la pérdida de aceite. En efecto, se ha calculado $\tau$ simulando el síndrome con diferentes niveles de incidencia; y se observa (Figura 5) que no es un indicador del síndrome tan sensible como la varianza o el sesgo. 


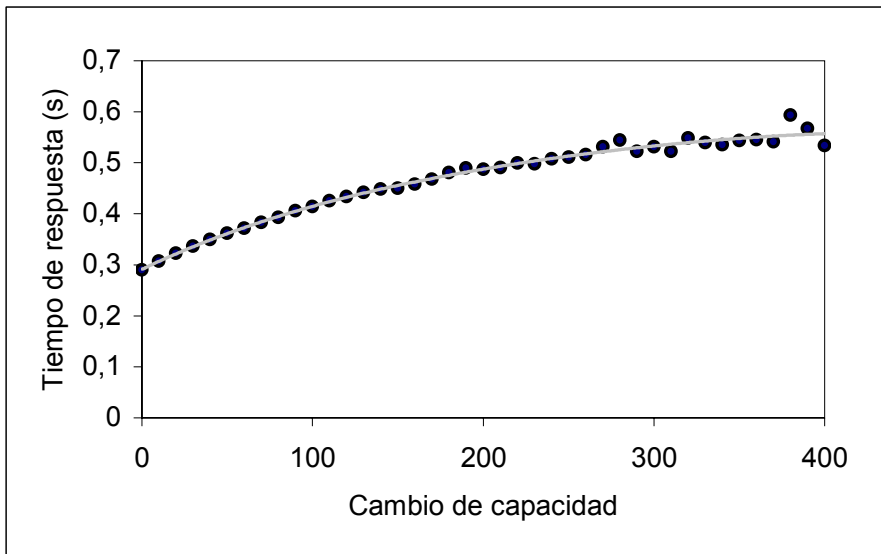

Fig. 5: Tiempo de respuesta, calculado suponiendo linealidad, en función del cambio de capacidad $\Delta C / C_{1}=\left(C_{2}-C_{1}\right) / C_{1}$ asociado a la presencia del síndrome.

Para realizar este análisis, en el caso del síndrome, puede procederse de una manera más simple: se propone descomponer la señal de ruido en sus partes positiva y negativa:

$x_{j}^{+}=\left\{\begin{array}{l}x_{j}, \text { si } x_{j} \geq 0 \\ 0, \text { si } x_{j}<0\end{array} \quad ; \quad x_{j}^{-}=\left\{\begin{array}{l}0, \text { si } x_{j} \geq 0 \\ x_{j}, \text { si } x_{j}<0\end{array}\right.\right.$

Ambas señales se han analizado como ruidos lineales, puesto que no son señales deterministas. Se han obtenido los tiempos de respuesta $\tau_{+}$y $\tau_{-}$correspondientes a cada una de ellas. Se ha llamado $a_{+}$al conjunto de coeficientes cuando el ruido es positivo y a cuando es negativo. Los coeficientes $a_{+}$toman el mismo valor que los a que se obtendrían si no existiera la pérdida de aceite. El sentido de este análisis se justifica porque un ruido lineal descompuesto según (3) da lugar a dos señales con coeficientes AR $a_{+}$y $a_{-}$iguales a los correspondientes al ruido lineal original.

Si se consideran los valores de la Tabla 1 para simular el comportamiento del sensor, y se supone una incidencia del síndrome tal que $C_{2} / C_{1}=2$, un posible conjunto de coeficientes $A R$ se recoge en la Tabla 2.

Si a partir de los coeficientes AR $a_{+}$y $a_{-}$se calcula la respuesta a la rampa positiva y negativa, se obtienen distintos valores, en conformidad con los valores determinados en las experiencias de laboratorio. Se han obtenido las respuestas a las rampas para $C_{2} / C_{1}=2$. Los tiempos de respuesta obtenidos son $\tau_{+}=0.24 \mathrm{~s}$, caso normal; y $\tau_{-}-=0.47 \mathrm{~s}$, caso de síndrome.

Tabla 2: Coeficientes AR: $a_{+}$corresponde al caso normal $\left(C_{2}=0\right)$ y $a_{-}$corresponde al caso de pérdida de aceite en el que $C_{2} / C_{1}=2$. Tiempo de muestreo $0.01 \mathrm{~s}$.

\begin{tabular}{|c|c|c|c|c|c|c|}
\hline & 1 & 2 & 3 & 4 & 5 & 6 \\
\hline$a_{+}$ & 2.035 & -2.489 & 2.156 & -1.010 & 0.351 & -0.075 \\
\hline$a_{-}$ & 2.048 & -2.497 & 2.168 & -1.010 & 0.348 & $-0,073$ \\
\hline
\end{tabular}


Puede obtenerse un índice dado por el cociente $\left|\tau_{-}-\tau_{+}\right| / \tau$, donde $\tau_{+}$y $\tau_{-}$son los tiempos de respuesta obtenidos descomponiendo la señal de ruido en sus partes positiva y negativa, respectivamente; y $\tau$ es el tiempo de respuesta considerando el ruido procedente de un sistema lineal. El índice así calculado es un indicador de la gravedad del síndrome. En la Figura 6 se muestra la representación del índice de anomalía en función del cambio de capacidad producido por el síndrome. Como puede observarse en la Figura 6, cuando este índice es mayor que $100 \%$ indica la presencia de la anomalía, ya que a partir del $100 \%$ cambia cualitativamente la distribución de los valores: primero se ajustan a una recta y después forman una nube en torno a una recta diferente.

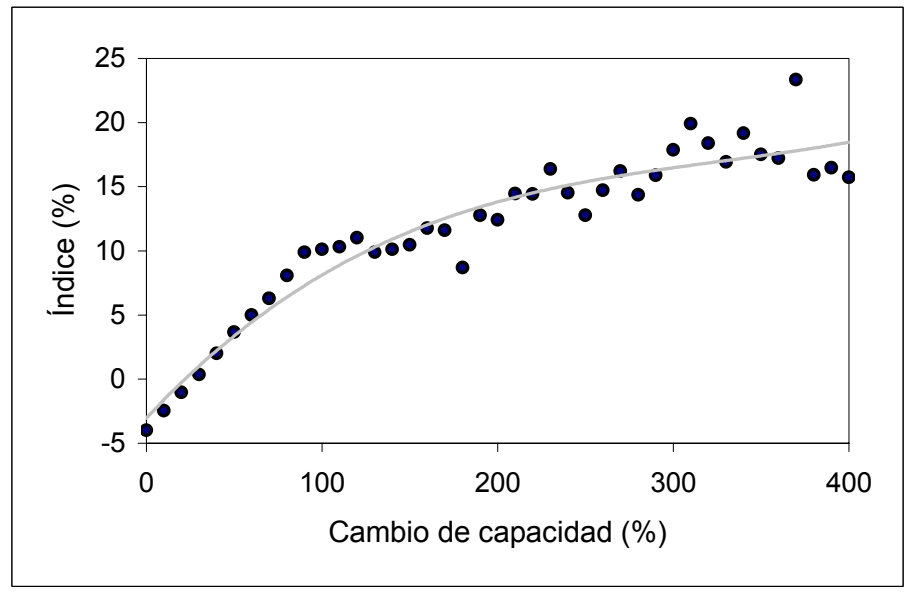

Fig. 6: Índice $\Delta \tau / \tau$ en función del cambio de capacidad $\Delta C / C_{1}=\left(C_{2}-C_{1}\right) / C_{1}$ asociado a la presencia del síndrome.

\section{CONCLUSIONES}

Se propone un modelo para explicar cuantitativamente el comportamiento bilineal observado en los sensores de presión del tipo Rosemount que presentan el síndrome de la pérdida de aceite. El modelo consiste en una analogía eléctrica con dos diodos para reproducir respuestas no lineales.

Se ha simulado la respuesta bilineal con dos modelos $A R$, de manera que cada uno de ellos proporcione la respuesta cuando actúe cada uno de los diodos. Se han simulado cuarenta respuestas lineales variando poco a poco la capacidad del sensor, así se ha podido detallar la evolución de la avería. Este procedimiento evita resolver numéricamente ecuaciones diferenciales estocásticas no lineales, y permite extender la aplicación de los modelos AR, que en el ámbito del mantenimiento son más conocidos que los métodos no lineales.

El análisis de ruido de las señales generadas reproduce los resultados experimentales observados tanto en el laboratorio como en las plantas. Se ha diagnosticado el síndrome a partir del decrecimiento de la varianza, y a partir del aumento del sesgo del ruido no lineal generado. También se ha comprobado que el tiempo de respuesta no es la magnitud de vigilancia adecuada para detectar la presencia incipiente del síndrome.

Considerando la asimetría del ruido bilineal, se ha propuesto un índice de vigilancia basado en el tiempo de respuesta, para cuantificar la intensidad de la anomalía. Este índice tiene la ventaja de calcularse con los algoritmos lineales que se emplean para medir el tiempo de respuesta, extendiéndose así la aplicación de una metodología lineal bien conocida al ámbito no lineal.

\section{REFERENCIAS}

Bergdahl, B.G., M. Morén y R. Oguma; Experiences from sensor tests and evaluation of sensor performance. Progress in Nuclear Energy: 43(1-4), 343-348 (2003). 
Barbero, J., J. Blázquez y O. Vela; Bubbles in the sensing line of Nuclear Power Plants pressure transmitters: the shift of spectrum resonances. Nuclear Engineering and Design: 199(3), 327-334 (2000).

Blázquez, J. y O. Vela; A useful model for the capacitive sensor oil-loss problem. Proceedings of the International Meeting on Reactor Noise (IMORN-27), 3-10, Valencia, España, 18 a 20 de Noviembre (1997).

García-Berrocal, A., J. Chicharro, M. Balbás y J. Blázquez; A fuzzy logic approach for noise signal reconstruction: Application to pressure sensors. Mechanical Systems and Signal Processing: 19(3), 501-508 (2005).

Glöckler, O., D.F. Cooke, G.J. Czuppon y K.K. Kpoor; Estimating the response time of pressure/flow transmitters and RTDs in CANDU reactors. Proceeding of the International Meeting on Reactor Noise (IMORN-28), 271-286, Atenas, Grecia, 11 a 13 de Octubre (2000).

Hashemian, H.M., D.W. Mitchell, R.E. Fain y K.M. Petersen; Long term performance and aging characteristics of Nuclear Plant pressure transmitters. Nuclear Regulatory Commission, NUREG/CR5851, Washington D.C., USA (1993).

Lin, K. y K.E. Holbert; Blockage diagnostics for nuclear power plant pressure transmitter sensing lines. Nuclear Engineering and Design: 239(2), 365-372 (2008).

Lin, K. y K.E. Holbert; Applying the equivalent pi circuit to the modeling of hydraulic pressurized lines. Mathematics and Computers in Simulation: 79(7), 2064-2075 (2009).

Ortiz-Villafuerte, J., R. Castillo-Durán, G. Alonso, y G. Calleros-Micheland; BWR online monitoring system based on noise analysis. Nuclear Engineering and Design: 236(22), 2394-2404 (2006).

Priestly, M.B.; Spectral Analysis and Time Series, pp 291-384, Academic Press Ltd, Londres, Inglaterra (1981).

Rosemount Eng. Company, Nuclear Operating Group, Status report on oil-loss Rosemount model 1153 series B/D and 1154 transmitters. Report No. D9200129, Minneapolis, USA (1992).

Vásquez, R.E. y F. Castrillón; Strategies to Compensate Non-Linearities Generated by Valves in Flow Control Loops. Información Tecnológica: 18(1), 161-168 (2007). 